\title{
Application of Electromagnetic Waves in Cancer Treatment by Hyperthermia
}

\author{
Md. Ziaul Amin \\ Electronics and Communication Engineering Discipline, Khulna University, Khulna, Bangladesh \\ Email: ziaulece@gmail.com \\ Received 24 April 2014; revised 21 May 2014; accepted 15 June 2014 \\ Copyright ( 2014 by author and Scientific Research Publishing Inc. \\ This work is licensed under the Creative Commons Attribution International License (CC BY). \\ http://creativecommons.org/licenses/by/4.0/ \\ (c) (i) Open Access
}

\begin{abstract}
Hyperthermia is a treatment mechanism by which cancer cells can be damaged. In this mode of treatment, electromagnetic fields are used for increasing the temperature of malignant tissues at sufficiently high level for destroying them. In this paper, major problems of hyperthermia and some proposed solutions are presented.
\end{abstract}

\section{Keywords}

\section{Hyperthermia, Electromagnetic Fields and Cancer}

\section{Introduction}

Hyperthermia means a body temperature that is higher than normal, which is caused due to failure of the heatregulating mechanisms of the body. But hyperthermia can also refer to heat treatment - the carefully controlled use of heat for medical purposes. In this treatment mechanism, controlled heat is applied to human malignant tissues for damaging them. In this method, temperature is usually raised up to $42^{\circ} \mathrm{C}-45^{\circ} \mathrm{C}$ and it can be induced by drugs, medical devices and externally applied electromagnetic fields. There are three different ways of application of this treatment-local, regional and whole body hyperthermia [1]. Local hyperthermia is used to heat small area like a tumor. On the other hand, large, deeply seated, and inoperable tumors are treated by regional hyperthermia. Whole-body hyperthermia is being used as a way to make chemotherapy work better in treating cancer. This potential cancer treatment technique can be used effectively, if the existing problems of this technique are solved.

\section{Problems of Hyperthermia}

The high energy application to human tissues could cause several problems. Firstly, it is difficult to administer 
heat treatment to a local region in the living body to attain the desired temperature in basic clinical or in vivo experiments [2] [3]. Secondly, measurement of internal body temperature non-invasively and precisely is not an easy task. Finally, uniform heating throughout the target volume to ensure that all cancerous tissues reach therapeutic temperature and achieve adequate temperature in deep tumors without damaging the surrounding healthy tissues is another serious engineering challenge. Therefore, major challenges in this treatment are to raise homogenous temperature, measure the internal temperature precisely and concentrate heat only on the affected tissues without damaging surrounding healthy tissues. To overcome these limitations different mechanisms have been proposed.

\section{Application of Nanoparticles in Hyperthermia}

In hyperthermia treatment, external applied electromagnetic field interacts with the electrical and magnetic properties of human cell. If the properties of the cells are modified by injecting suitable particles for increasing heating and focusing effect of induced temperature, the effectiveness of this treatment can be increased significantly. For increasing the heat concentration in the targeted cells, nanoparticle insertion technique is proposed in [4]. In this proposed technique magnetic properties of the injected materials are used to convert applied electromagnetic field energy into heat. This transformation of the magnetic energy into heat is due to the N'eel relaxation, which is caused by the rapidly alternating magnetic dipole moments, and the Brownian relaxation, which is due to nanoparticle rotation, resulting in friction of the particles with the fluid. As we know the magnetic fields can penetrate the body and interact with the body tissues. Therefore, the application of an external electromagnetic (EM) field in the desired range of radio frequencies will generate a thermal shock in the target area which will destroy the cells of the tumor without damaging the surrounding healthy tissues noninvasively. Actually, magnetic nanoparticles are used for increasing the susceptibility of the carcinogenic cells for increasing focusing effect of heat.

In summary, hyperthermia treatment by using nanoparticle can be divided into three steps:

1) Feeding magnetic fluid to tumor or cancer;

2) Applying external electromagnetic fields;

3) Tumor or cancer is destroyed (apoptosis).

Treatment procedure of cancer cells by nanoparticle is shown in the Figure 1.

\section{Non-Invasive Temperature Measurement}

Appropriate temperature measurement in the affected area is very important for avoiding healthy tissue damage due to overheating. In the non-invasive temperature measurement technique, target material dielectric constant is measured from the resonant frequency of the cavity generator [3]. Then the temperature dependence of the dielectric constant for some materials can then be estimated by observing the resonant frequency change with temperature. For example, the temperature dependence of the dielectric constant of pure water is given by (1).

$$
\varepsilon_{r} \approx 87.74-0.40 T+9.40 \times 10^{-4} T^{2}-1.41 \times 10^{-6} T^{3}
$$

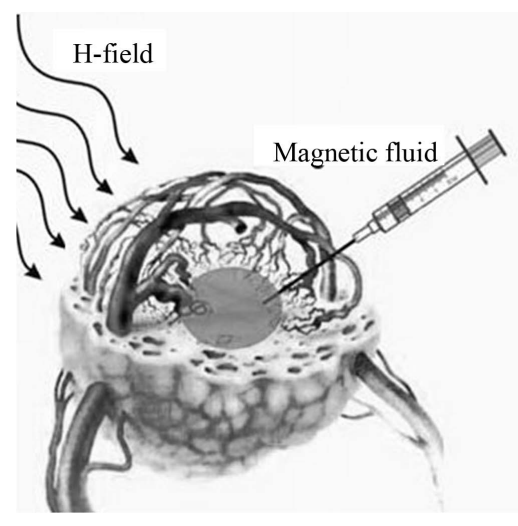

Figure 1. Cancer treatment by using magnetic nanoparticles. 


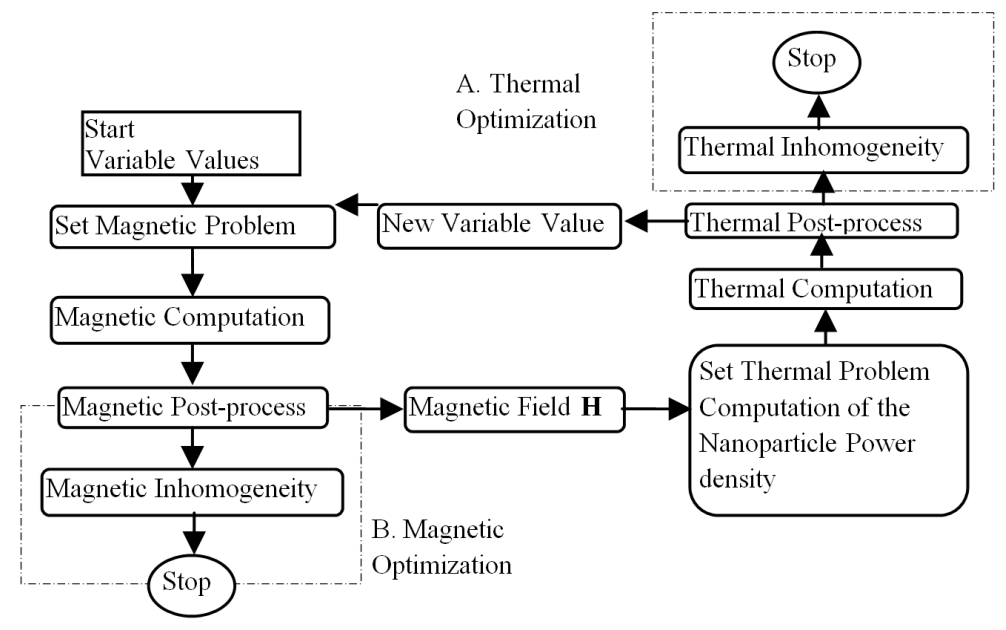

Figure 2. Flow chart for thermal and magnetic coupled uniformity search.

Here, $T, \varepsilon_{r}$ represents the temperature in $\left({ }^{\circ} \mathrm{C}\right)$ and relative dielectric constant of material respectively.

However, since it is necessary to measure a frequency change of only tens of kilohertz, in contrast to a resonant frequency of hundreds of megahertz, this measurement is particularly difficult when a living body is the target. In such cases, the frequency change with temperature cannot be measured directly; furthermore, the spatial frequency change cannot be distinguished. But phase change due to a frequency change may be detected, which improves the measurement accuracy and provides spatial information. In Equation (2), the minute frequency change with temperature can be detected as an expanded phase change in the electromagnetic waves (i.e. $\nabla \theta$ ) by adjusting the observation time.

$$
\nabla \theta((T(r))) \approx 2 \pi\left[f(T(r))-f\left(T_{0}(r)\right)\right] t_{\text {delay }}
$$

$T_{0}, r$, and $t_{\text {delay }}$ represent the reference temperature $\left({ }^{\circ} \mathrm{C}\right)$, spatial vector, and time delays with respect to a reference time (s), respectively.

Therefore, temperature change inside an object can be detected by observing the phase change distribution accompanying the associated dielectric constant change in a cavity resonator. The proposed method can be easily embedded in a heating applicator based on a cavity, so a novel cancer treatment system that combines localized heating of cancers with non-invasive temperature monitoring could be established.

\section{Uniform Field and Temperature}

Uniform heating of the affected area is the most important for damaging the cancer tissues and keeping the healthy tissues unaffected. For maintaining uniform heating magnetic field uniformity is required. Figure 2 shows the thermal and magnetic coupled optimization to search magnetic and thermal uniformity [5].

\section{Conclusion}

Hyperthermia is a promising way to improve cancer treatment, but it is largely an experimental technique at this time and is not commonly used. Research is going on to better understand this technique and improve the treatment efficiency. For instance, the use of nanoparticles, non-invasive temperature measurement and uniform heating techniques is some recent studies which provide better results than existing hyperthermia. For the best use of this method, researchers continue to investigate new approach.

\section{References}

[1] Sardari, D. and Verga, N. (2011) Cancer Treatment with Hyperthermia. http://cdn.intechopen.com/pdfs/24593/InTech-Cancer_treatment_with_hyperthermia.pdf

[2] Szigeti, G.P., Hegyi, G. and Szasz, O. (2012) Hyperthermia versus Oncothermia: Cellular Effects in Cancer Therapy. Conference of the International Clinical Hyperthermia Society, 2013, Article ID: 274687. 
[3] Ishihara, Y. and Ohwad, H. (2011) Non-Invasive Temperature Measurement by Using Phase Changes in Electromagnetic Waves in a Cavity Resonator. International Journal of Hyperthermia, 27, 726-736. http://dx.doi.org/10.3109/02656736.2011.602038

[4] Trujillo-Romero, C.J., Garc'1a-Jimeno, S., Vera, A., Leija, L. and Estelrich, J. (2011) Using Nanoparticles for Enhancing the Focusing Heating Effect of an External Waveguide Applicator for Oncology Hyperthermia: Evaluation in Muscle and Tumor. Progress in Electromagnetics Research, 121, 343-363. http://dx.doi.org/10.2528/PIER11092911

[5] Biomedical Applications of Electromagnetic Fields: Human Exposure, Hyperthermiaand Cellular Stimulation. http://paduaresearch.cab.unipd.it/4040/1/tesi_rev67_finale2.pdf 